\title{
Schulöffnung in Sars-CoV-2-Zeiten
}

In Deutschland haben die Schulen in den meisten Bundesländern bereits Anfang und Mitte August wieder ihren Betrieb aufgenommen. Wegen der Besorgnis in Sachen SARS-CoV-2-Infektionen hat die Ad-hocKommission SARS-CoV-2 der Deutschen Gesellschaft für Virologie (GfV) eine entsprechende Stellungnahme zu den Präventionsmaßnahmen abgegeben.

In der Stellungnahme werden alle Maßnahmen begrüßt, die dazu dienen, die Schulen und Bildungseinrichtungen in der kommenden Wintersaison offen zu halten. Es wird darauf hingewiesen, dass „nach heutigem Wissensstand Infektionen mit SARS-CoV-2 bei Kindern in der überwiegenden Mehrheit mild verlaufen, mit deutlich geringeren Raten an Hospitalisierung, Komplikationen und Todesfällen als bei Erwachsenen." Ein geregelter Schulbetrieb sei, so die Stellungnahme, nicht nur für die Entlastung berufstätiger Eltern, sondern auch für das Wohlergehen der Kinder von großer Bedeutung. Der Schulbetrieb müsse jedoch an pragmatische Konzepte gekoppelt sein, die das Risiko der Infektionsausbreitung an Schulen eliminieren oder zumindest deutlich reduzieren können. Denn, "für eine wirksame Unterdrückung der Virusausbreitung in der Gesamtgesellschaft bleibt es auch wei-

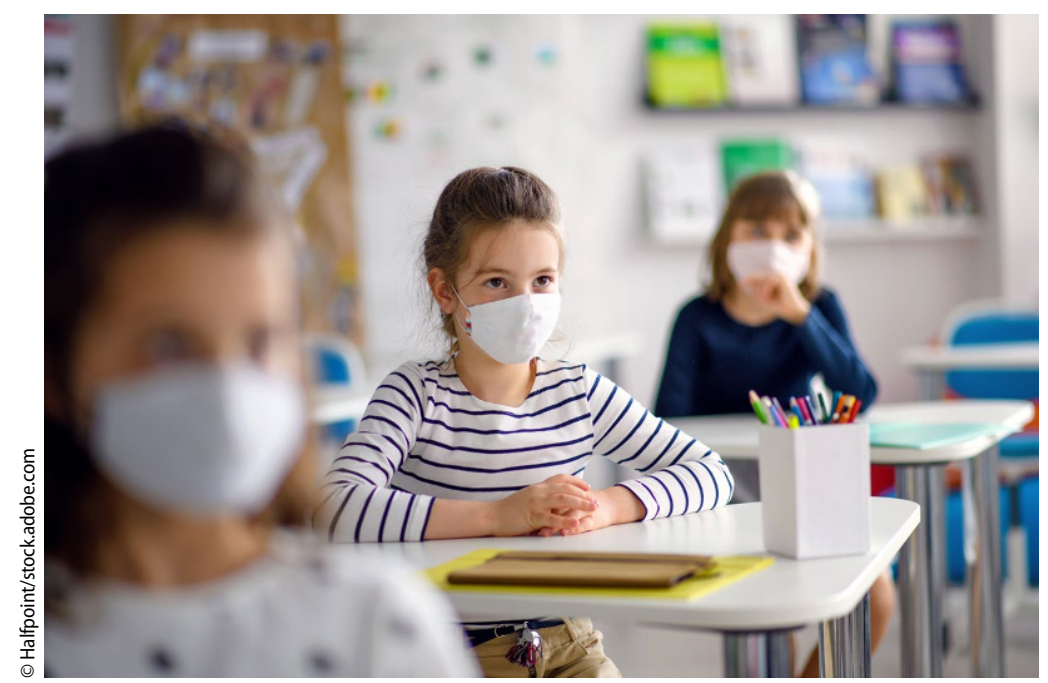

$\Delta$ Die Deutsche Gesellschaft für Virologie empfiehlt das Tragen des Mund-Nasen-Schutzes konsequent in allen Altersstufen terhin eine Grundvoraussetzung, die Viruszirkulation in den Schulen niedrig zu halten." Eine effektive Kontrolle der Neuinfektionen in der Umgebung der Schulen, also dem privaten Umfeld von Schülern und Lehrkräften, sei die beste Prävention für die Eintragung des Virus in die Schulen.

\section{Umfassende Studien fehlen bisher} Ausdrücklich warnen die Experten der GfV davor, zu glauben, dass Kinder keine Rolle in der Pandemie und in der Übertragung spielen. Dies stehe nicht im Einklang mit den wissenschaftlichen Erkenntnissen. „Fehlende Präventions- und Kontrollmaßnahmen könnten in kurzer Zeit zu Ausbrüchen führen, die dann erneute Schulschließungen erzwingen." Umfassende wissenschaftliche Studien über die Infektionsraten bei Kindern und deren Rolle in der Pandemie liegen bisher nicht vor. Zunächst hatte man angenommen,

dass Kinder in der Infektionskette nur eine geringe Rolle spielen. Nun weisen neuere wissenschaftliche Veröffentlichungen und konkrete Beobachtungen in einigen Ländern darauf hin, dass dies in Frage gestellt werden müsse. Frühere Studien seien überwiegend unter den Bedingungen des "Lockdown" mit Schulschließungen und reduzierten Kontakten durchgeführt worden. Dies entspreche nicht den Rahmenbedingungen eines wieder im angepassten Regelbetrieb laufenden Schul- und Alltagslebens. Der prozentuale Anteil von Kindern an der Gesamtzahl der Neuinfektionen in Deutschland liege, so die Virologen, in einer Größenordnung, die dem Anteil der Kinder an der Gesamtbevölkerung entspreche [1].

Schwierig sei weiterhin die Interpretation der Daten zur eigentlichen Übertragungshäufigkeit bei Kindern im Vergleich zu Erwachsenen. Ergebnisse aus einigen sorgfältig durchgeführten Haushaltsstudien zeigten, dass Kinder etwa gleich häufig infiziert waren wie Erwachsene [2, 3]. Wie oft Kinder das Virus nun tatsächlich übertragen bleibe einstweilen unklar, wobei eine neue umfassende Beobachtungsstudie aus Italien nahe lege, dass Kinder Infektionen in erhöhtem Maße übertragen. Die Autoren führen dies auf deren intensiveres Kontaktverhalten zurück [4]. Dagegen zeigt eine Studie von Zhang et al., dass Kinder, trotz höherer angenommener Kontaktraten, eine etwa gleich hohe Infektionshäufigkeit wie Erwachsene hatten. Demnach haben Kinder eine geringere Empfänglichkeit [3]. Die meisten Studien stammen aus Haushaltsbeobachtungen, nur 
wenige Daten liegen aus der tatsächlichen Schulsituation vor. Beispiele von tatsächlichen SARS-CoV-2-Clustern an Schulen in Israel und Australien untermauern jedoch das gegebene Risiko von Ausbruchsgeschehen im Bildungsbereich, insbesondere bei einem verstärkten Gesamt-Infektionsgeschehen in der Bevölkerung $[5,6]$.

\section{Aerosolübertragung unterschätzt}

Eine der wichtigen neuen Erkenntnisse zu SARS-CoV-2, die bei der Schulöffnung bedacht werden müssen, betrifft die inzwischen anerkannte Möglichkeit der Aerosolübertragung, also die Übertragung durch die Luft, insbesondere in Innenräumen bei unzureichender Luftzirkulation [7]. Je mehr Personen sich in einem geschlossenen Raum befinden und je länger die dort verbrachte Zeitspanne ist, desto größer ist das Risiko einer Über- tragung. Eine Situation, die auf den Schulbetrieb passgenau zutrifft. Kleingruppen, digitale Lösungen, Durchlüftung, Mund-Nasen-Schutz, Händehygiene und ähnliche Maßnahmen sollten daher strukturiert und konsequent flächendeckend durchgeführt werden.

Aus alleiniger virologischer Sicht spricht sich das Expertenteam der GfV für das konsequente Tragen von Alltagsmasken in allen Schuljahrgängen auch während des Unterrichts aus. Ebenso sollte eine konsequente Händehygiene beibehalten werden, auch wenn die Übertragung durch Oberflächen wahrscheinlich initial überschätzt und die aerogene Übertragung unterschätzt wurde.

\section{Die gesamte Stellungnahme}

im Internet: www.g-f-v.org/

stellungnahmen_detail

\section{Literatur}

1. https://www.rki.de/DE/Content/ InfAZ/N/Neuartiges_Coronavirus/

Situationsberichte/2020-07-21-de.pdf? blob=publicationFile

2. Bi et al (2020) Lancet Infect Dis 20(8):911-919

3. Zhang et al (2020) Science 26:1481-1486

4. Fateh-Moghadam et al medRxiv 2020.07.16.20127357

5. Stein-Zamir et al (2020) Euro Surveill 25(29)

6. https://www.dhhs.vic.gov.au/coronavirusupdate-victoria-thursday-16-july-2020

7. https://www.who.int/news-room/commentaries/detail/transmission-of-sars-cov-2-implications-for-infection-prevention-precautions

Hinweis des Verlags. Der Verlag bleibt in Hinblick auf geografische Zuordnungen und Gebietsbezeichnungen in veröf-

fentlichten Karten und Institutsadressen neutral.

Wien klin Mag 2020 · 23:184-185 https://doi.org/10.1007/s00740-02000359-9

(c) Springer-Verlag GmbH Austria, ein Teil von Springer Nature 2020
Quelle: Presseaussendung Deutsche Gesellschaft für Virologie

Hier steht eine Anzeige. 